Supporting Information for

\title{
High Thermopower in a Zn-based 3D Semiconductive Metal-Organic Framework
}

Jihye Park ${ }^{1, \dagger}$, Allison C. Hinckley ${ }^{1, \dagger}$, Zhehao Huang ${ }^{2, *}$, Gan $\mathrm{Chen}^{3}$, Andrey A. Yakovenko ${ }^{4}$, Xiaodong $\mathrm{Zou}^{2}$, Zhenan Bao ${ }^{1, *}$

${ }^{1}$ Department of Chemical Engineering, Stanford University, Stanford, CA 94305, USA

${ }^{2}$ Berzelii Centre EXSELENT on Porous Materials, Department of Materials and Environmental Chemistry, Stockholm University, SE-106 91 Stockholm, Sweden

${ }^{3}$ Department of Materials Science and Engineering, Stanford University, Stanford, CA 94305, USA

${ }^{4}$ X-ray Science Division, Advanced Photon Source, Argonne National Laboratory, Argonne, IL 60439, USA

$\dagger$ These authors equally contributed.

\section{Table of Contents:}

Section 1. Materials and instrumentation

Section 2. Zn-HAB synthesis and characterizations

Section 3. Structure determination

Section 4. Electrical measurements 


\section{Section 1. Materials and instrumentation}

All reagents and starting materials were purchased from Sigma-Aldrich or TCI and used without further purification. Hexaaminobenzene (HAB) was synthesized according to the literature procedure. ${ }^{\mathrm{S} 1}$ Powder X-ray diffraction was carried out on a BRUKER D8 Venture Single Crystal Diffractometer equipped with a Cu-sealed tube $(\lambda=1.54 \AA)$ using a capillary at $50 \mathrm{kV}$ and $1 \mathrm{~mA}$. Synchrotron powder X-ray diffraction was carried out on the 17-BM at the Advanced Photon Source $(\lambda=0.45260 \AA)$, Argonne National Laboratory, IL, USA. Scanning electron microscopy (SEM) analysis was performed on a field emission-scanning electron microscope (Magellan 400 XHR) at 3 $\mathrm{kV}$. Absorption spectra were taken on a CARY 6000i UV-Vis-NIR spectrophotometer. To prepare the UV-Vis sample, as-synthesized $\mathrm{Zn}-\mathrm{HAB}(\sim 5 \mathrm{mg})$ was washed with $\mathrm{H}_{2} \mathrm{O}(3 \times 2 \mathrm{~mL})$, centrifuged, and then the wet sample was re-dispersed in isopropyl alcohol and drop-casted on a glass substrate for UVVis measurement after drying. Gas adsorption measurements were measured using an Autosorb IQ2. The powder samples were dried at the set temperature for $2 \mathrm{~h}$ for the sorption measurements. Electrical conductivity measurements were carried out using a Keithley 4200 SCS parameter analyzer. Nicolet iS50 FT-IR spectrometer was used for IR spectra. Riken AC-2 photoelectron spectrometer was used for PESA measurements. X-ray photoelectron spectroscopy (XPS) was performed using PHI Versaprobe III with an $\mathrm{Al} \mathrm{Ka}$ radiation. 


\section{Section 2. Zn-HAB synthesis and characterizations}

\section{$\mathrm{NH}_{4} \mathrm{OH}$ as the base}

$22.0 \mathrm{mg}$ of zinc nitrate hexahydrate $\left[\mathrm{Zn}\left(\mathrm{NO}_{3}\right)_{2} \cdot 6 \mathrm{H}_{2} \mathrm{O}\right]$ was dissolved in $10 \mathrm{~mL}$ of $\mathrm{H}_{2} \mathrm{O} .13 .8 \mathrm{mg}$ of $\mathrm{HAB} \cdot 3 \mathrm{HCl}$ in an additional $5 \mathrm{~mL}$ of $\mathrm{H}_{2} \mathrm{O}$ was then added to the zinc nitrate solution while stirring at $300 \mathrm{rpm} .82 .8 \mu \mathrm{L}$ of $\mathrm{NH}_{4} \mathrm{OH}(6 \mathrm{M})(10$ equiv. to $\mathrm{HAB})$ was then added. The mixture was stirred for 2 $h$ at $R T$ in air. The resulting dark red solids were filtered and washed with $\mathrm{H}_{2} \mathrm{O}(2 \times 80 \mathrm{~mL})$ and acetone $(2 \times 50 \mathrm{~mL})$ and dried under vacuum for $1 \mathrm{~h}$ at $\mathrm{RT}$ for further characterization unless otherwise noted.

\section{Triethylamine (TEA) as the base}

$22.0 \mathrm{mg}$ of zinc nitrate hexahydrate $\left[\mathrm{Zn}\left(\mathrm{NO}_{3}\right)_{2} \cdot 6 \mathrm{H}_{2} \mathrm{O}\right]$ was dissolved in $7.5 \mathrm{~mL}$ of DMF. $13.8 \mathrm{mg}$ of $\mathrm{HAB} \cdot 3 \mathrm{HCl}$ in $7.5 \mathrm{~mL}$ of $\mathrm{H}_{2} \mathrm{O}$ was then added to the zinc nitrate solution while stirring at $300 \mathrm{rpm}$. $62.5 \mu \mathrm{L}$ of trimethylamine (TEA) (9 equiv. to HAB) was then added. The mixture was stirred for $2 \mathrm{~h}$ at RT in air. The resulting dark red solids were filtered and washed with $\mathrm{H}_{2} \mathrm{O}(2 \times 80 \mathrm{~mL})$ and acetone $(2 \times 50 \mathrm{~mL})$ and dried under vacuum for $1 \mathrm{~h}$ at $\mathrm{RT}$ for further characterizations unless otherwise noted. Different synthetic conditions were also examined by varying the amount of TEA to 3 equiv. and 6 equiv. relative to $\mathrm{HAB}$ with all the other conditions unchanged.

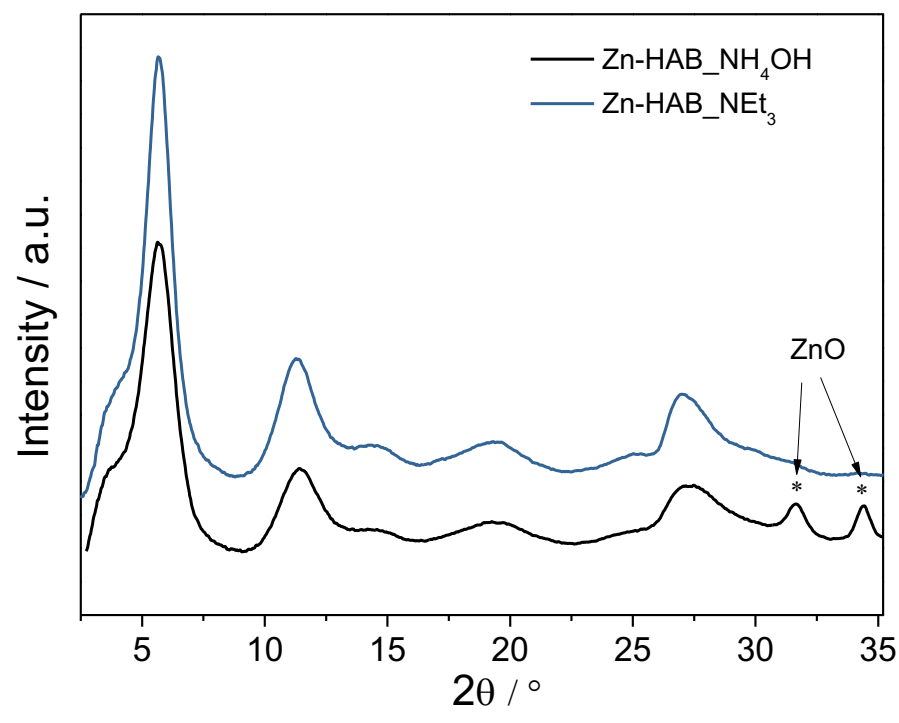

Figure S1 | PXRD of Zn-HAB products synthesized under different bases. 


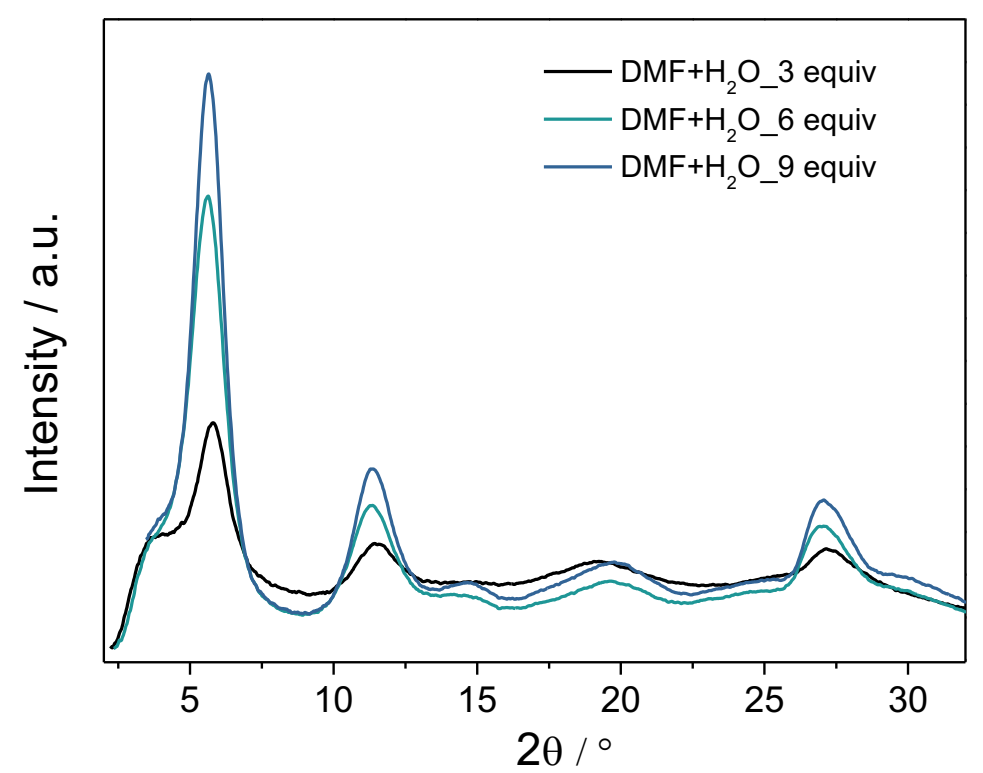

Figure S2 | PXRD of Zn-HAB products synthesized with different stoichiometries of triethylamine.

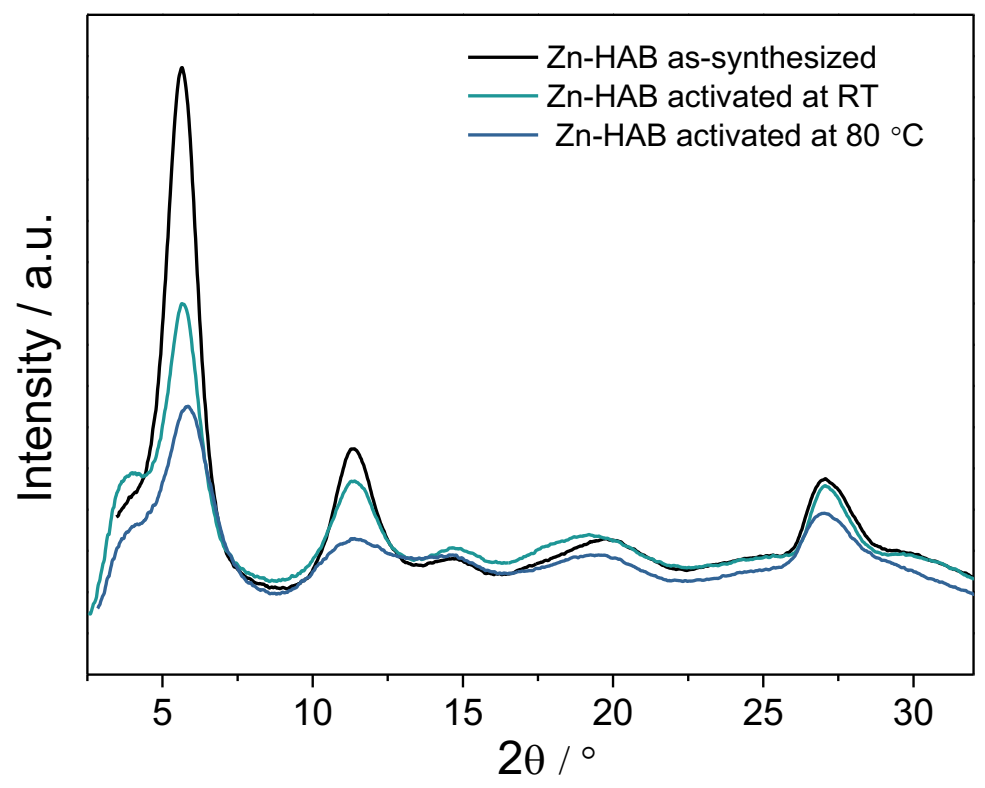

Figure S3 | PXRD of Zn-HAB samples after different activation treatments. 


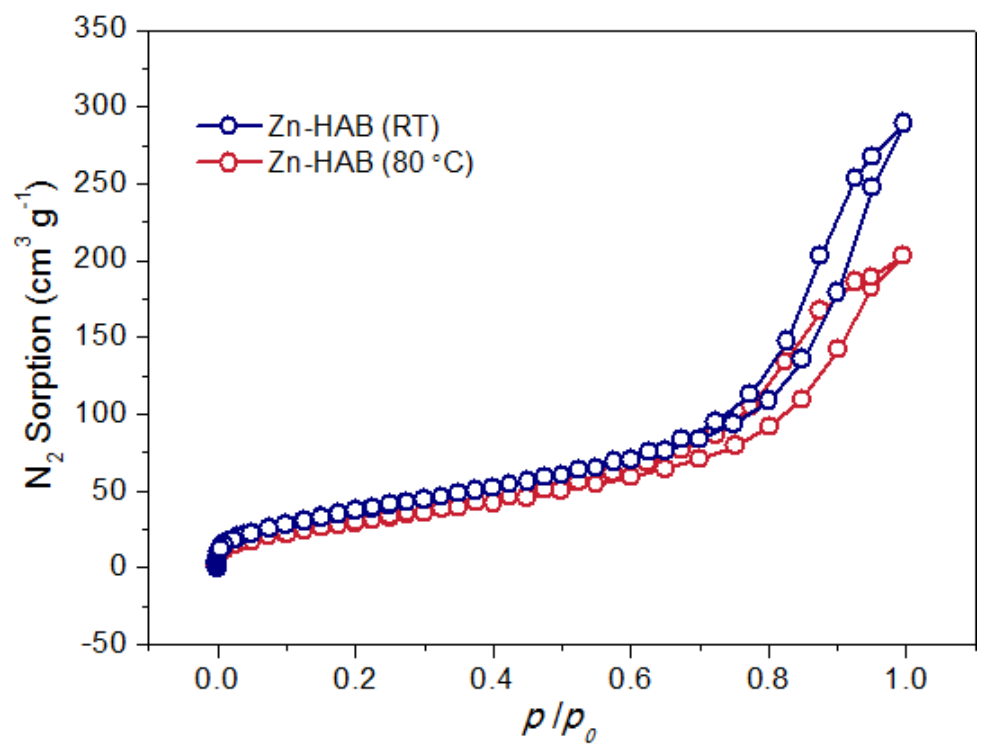

Figure S4 | $\mathrm{N}_{2}$ sorption isotherms of Zn-HAB samples after different treatments. The BET surface area of the samples decreases from $145 \mathrm{~m}^{2} / \mathrm{g}$ (RT), to $117 \mathrm{~m}^{2} / \mathrm{g}\left(80^{\circ} \mathrm{C}\right)$, suggesting collapse of the frameworks.

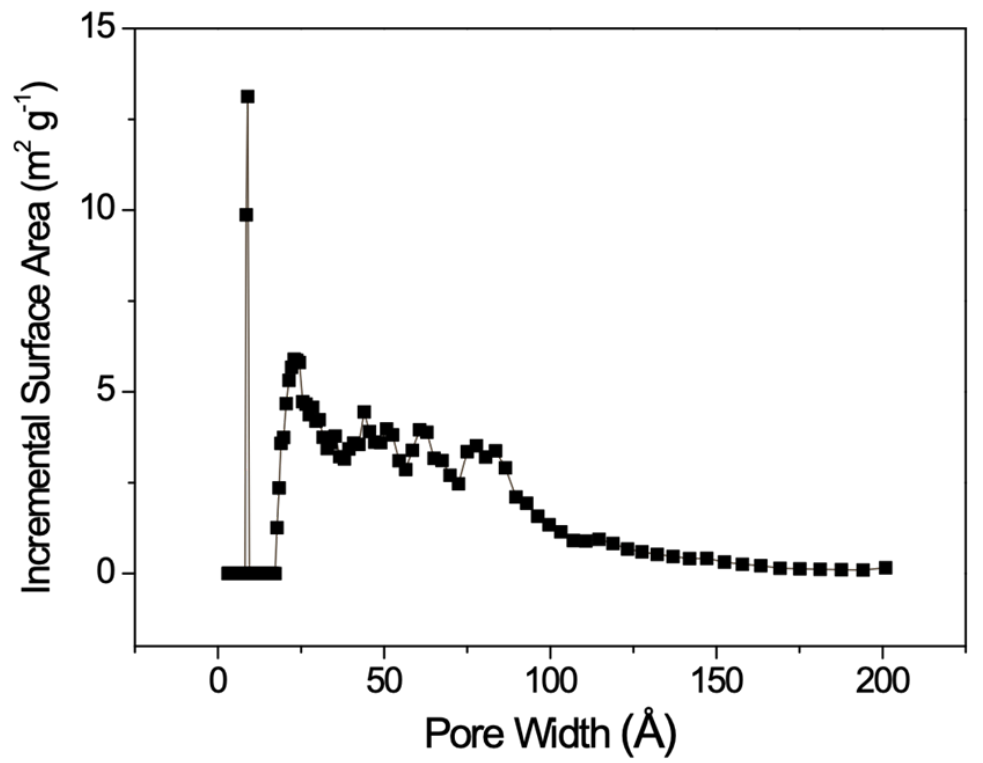

Figure S5 | Pore size distribution of Zn-HAB. 


\section{Section 3. Structural determination}

The analysis of the geometry of Zn-HAB was conducted using a pseudopotential plane-wave method within the DFT framework by Accelrys Materials Studio package. After creation of the initial structure, the geometry was optimized in the FORCITE module with the Dreiding forcefield of Materials Studio. The DFT calculations were performed in the generalized gradient approximation with the Perdew-Burke-Ernzerhof functional implemented in the CASTEP module of Accelrys Materials Studio. A kinetic energy cutoff of $400 \mathrm{eV}$ and a $\Gamma$-centered k-point mesh of $2 \times 2 \times 8$ was used for the calculations. A Pawley fit against the synchrotron PXRD data was further applied to refine the unit cell parameters, which was performed using TOPAS Academic V5.0. ${ }^{[\mathrm{S} 2]}$ The background curve was fitted with an 8th order Chebyschev polynomial. The fitting was conducted in space group $I 4_{1} 22$, using a Pearson VII type peak profile function followed by refinement of the unit cell.

Table S1. Crystallographic data, conditions of powder X-ray diffraction data collection, and Pawley fit results of Zn-HAB.

\begin{tabular}{ll}
\hline \multicolumn{1}{c}{ Name } & Zn-HAB \\
\hline Chemical formula & $\mathrm{C}_{24} \mathrm{~N}_{24} \mathrm{Zn}_{6}$ \\
Formula weight & 237.5 \\
Crystal system & Tetragonal \\
Space group & $I 4_{12} 2$ \\
$a / \AA$ & $21.7(1)$ \\
$c / \AA$ & $23.5(3)$ \\
$Z$ & 2 \\
Temperature/K & $298(2)$ \\
Wavelength $/ \AA$ & 0.45260 \\
$2 \theta$ range/ & $1.198708-18.123148$ \\
$R_{p}$ & 0.00389 \\
$R_{w p}$ & 0.00700 \\
$R_{\exp }$ & 0.00745 \\
GOF & 0.939 \\
\hline
\end{tabular}

Due to the small crystal size and broad peaks in PXRD pattern, ab initio structural determination by X-ray diffraction is not possible. Furthermore, the sample is not stable in TEM. Therefore, we have evaluated different structural models of $\mathrm{Zn}-\mathrm{HAB}$, including 2D models with $\mathrm{Zn}$ atoms are square planar coordinated, 3D models with $\mathrm{Zn}$ atoms are tetrahedrally coordinated, and models with a mixed tetrahedral and square planar coordination geometries. PXRD patterns of different 2D models were simulated, including AA-eclipsed, AA-inclined, AB-staggered and ABpartial staggered structures (Figure S6). The 2D structures show the first peak at ca $2.2^{\circ}$, which indicate a $d_{100}$ of around $11.50 \AA$. This is much smaller than the first peak in the observed PXRD pattern. Thus, the $2 \mathrm{D}$ structures were ruled out. Because the HAB linker has a $\mathrm{D}_{3 \mathrm{~h}}$ symmetry, and the triangles are the only component of the net of $\mathrm{Zn}-\mathrm{HAB}$, the srs net is the only regular net which contains only triangles. We then modeled different 3D structures of $\mathrm{Zn}-\mathrm{HAB}$ based on the srs net, including the srs net without interpenetration, the srs net with an interpenetration, and the srs net with a displaced interpenetration. Among them, the srs net without interpenetration agrees best to the observed PXRD 
pattern (Figure S7). However, crystals in a cubic unit cell is unlikely to grow anisotropically and form a rod shape, which is shown by SEM images. Thus, the symmetry of Zn-HAB was lowered to tetragonal while keep the srs topology. Taking preferred orientation into account, the second strongest peak in the simulated and experimental PXRD patterns shows better agreement (Figure S8). It is worth noting that the simulation is made from prefect crystal models, while the $\mathrm{Zn}-\mathrm{HAB}$ material has a high degree of disorder as indicated by the broad PXRD peaks. In addition, we cannot exclude the presence of mixed phases with all of the three srs nets. We further considered structural models with a mixed square planar and tetrahedral coordination geometry of Zn atoms (Figure S11). Nevertheless, a mixed coordination geometry results in larger unit cell dimensions and pore sizes. According to our observation of PXRD pattern (Figure S7) and porosity analysis, we can exclude the possibility of a mixed square planar and tetrahedral coordination geometry of $\mathrm{Zn}$ atoms.

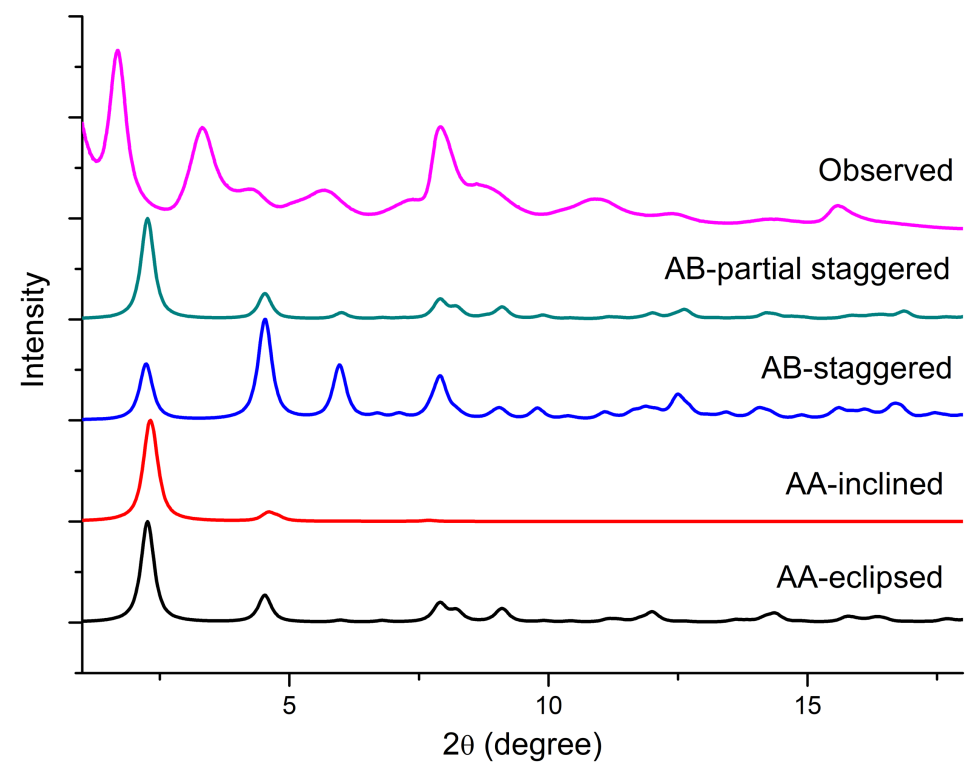

Figure S6 | Simulated PXRD patterns of Zn-HAB hypothetical models with 2D structures $(\lambda=$ $0.45260 \AA$ ). The PXRD patterns were simulated using a pseudo-Voigt peak shape function.

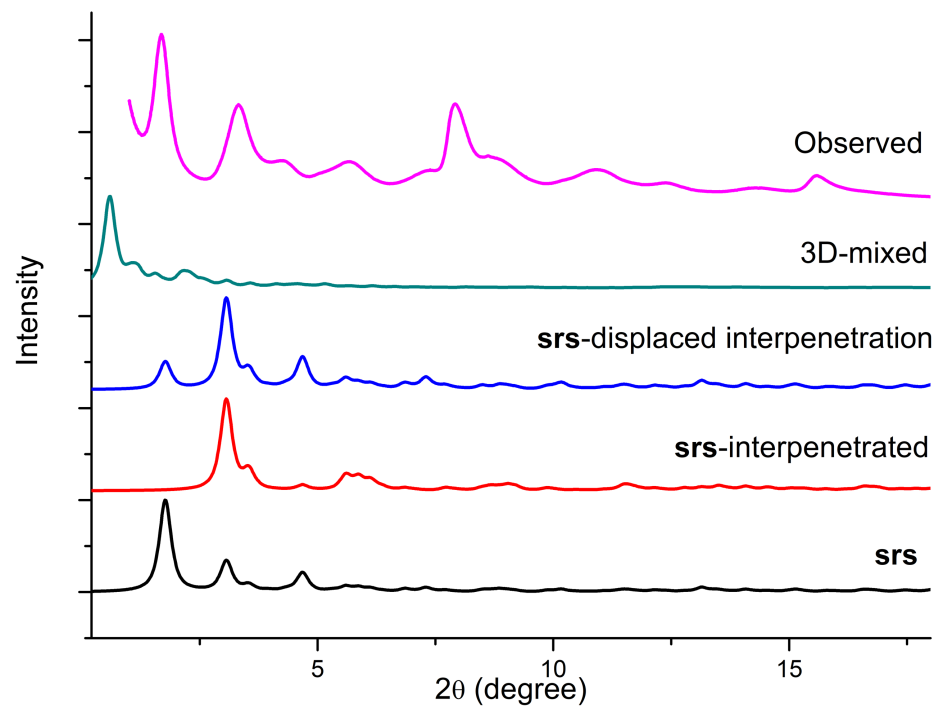

Figure S7 | Simulated PXRD patterns of Zn-HAB hypothetical models with 3D structures $(\lambda=$ $0.45260 \AA$ ). The PXRD patterns were simulated using a pseudo-Voigt peak shape function. 

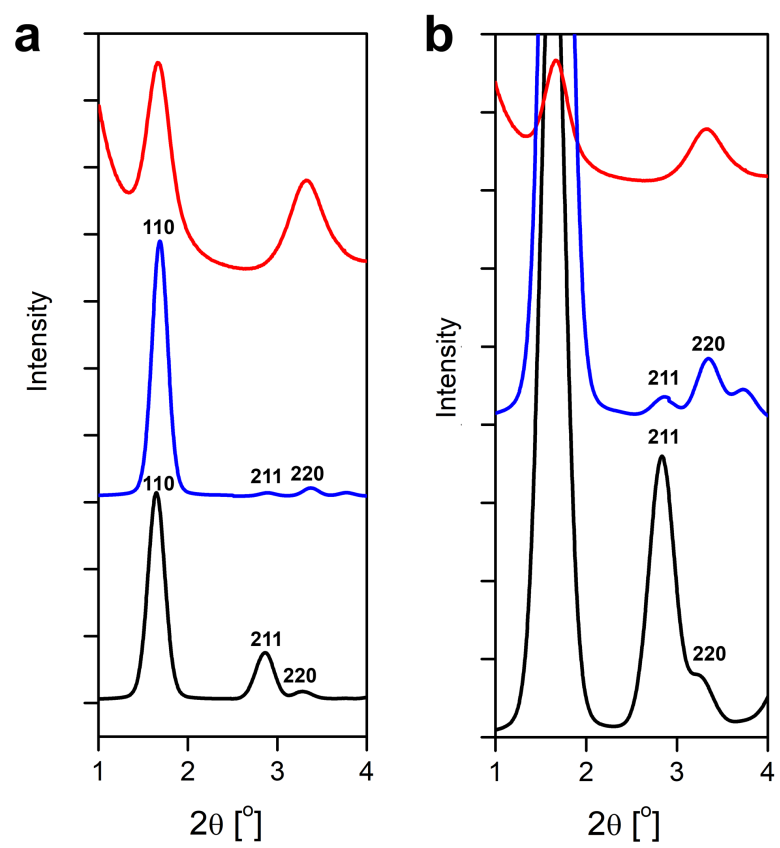

Figure S8 | Comparison of simulated PXRD patterns of Zn-HAB (black), simulated PXRD pattern of $\mathrm{Zn}$-HAB, with rod-shape preferred orientation applied along $c$-axis (blue), and experimental PXRD pattern $(\lambda=0.45260 \AA$, red) of Zn-HAB. (a) PXRD patterns normalized by the 110 reflection. (b) PXRD patterns normalized by the 211 reflection. Asterisk indicates the peak suppressed in the experimental pattern, which can be caused by preferred orientation of the rod-shape crystals. The PXRD patterns were simulated using a pseudo-Voigt peak shape function, and the preferred orientation was simulated using a weighted March-type correction with alignment along rod direction [001]. The much lower ratio between intensities of the 110 and 220 peaks in the experimental PXRD pattern is caused by the guest-molecules in the pores of $\mathrm{Zn}-\mathrm{HAB}$, which is not include in the structural model used for simulation of the PXRD pattern. 

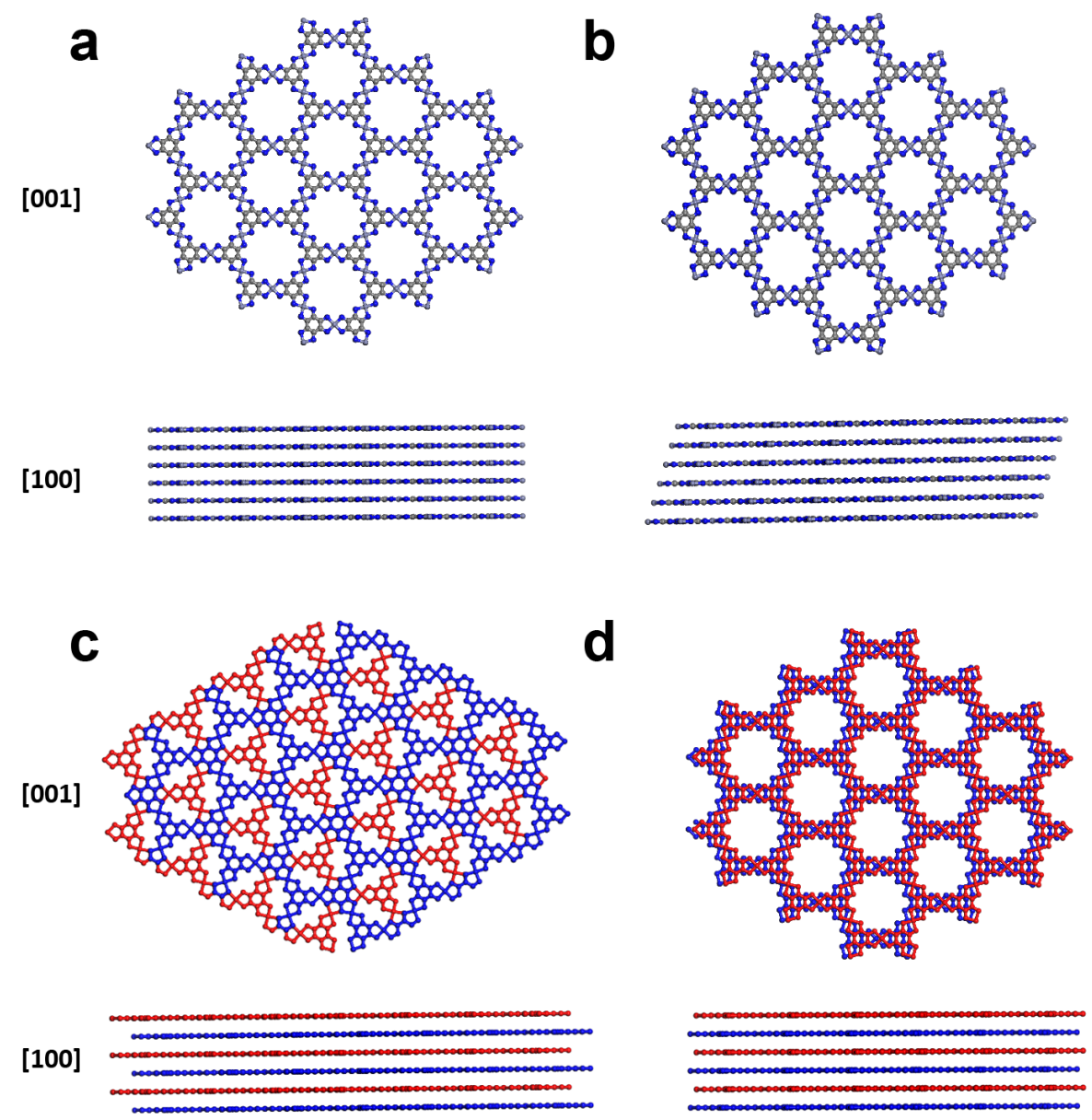

Figure S9 | Hypothetical models of Zn-HAB with 2D structures. (a) AA-eclipsed stacking. (b) AAinclined stacking. (c) AB-staggered stacking. (d) AB-partial staggered stacking. 

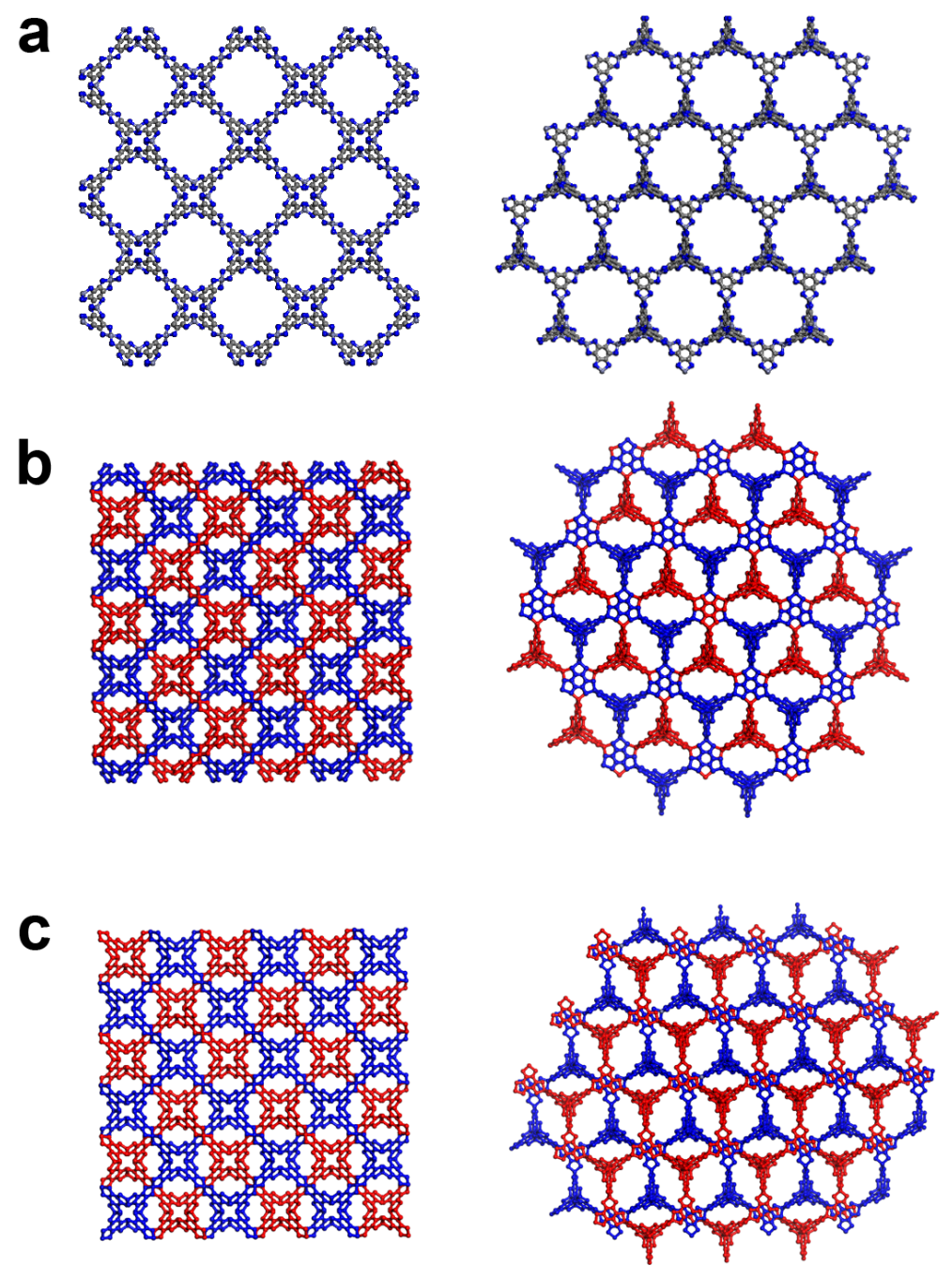

[100]

[111]

Figure S10 | Hypothetical models of Zn-HAB with 3D structures. (a) The srs net. (b) The srs net with an interpenetration. (c) The srs net with a displaced interpenetration. 

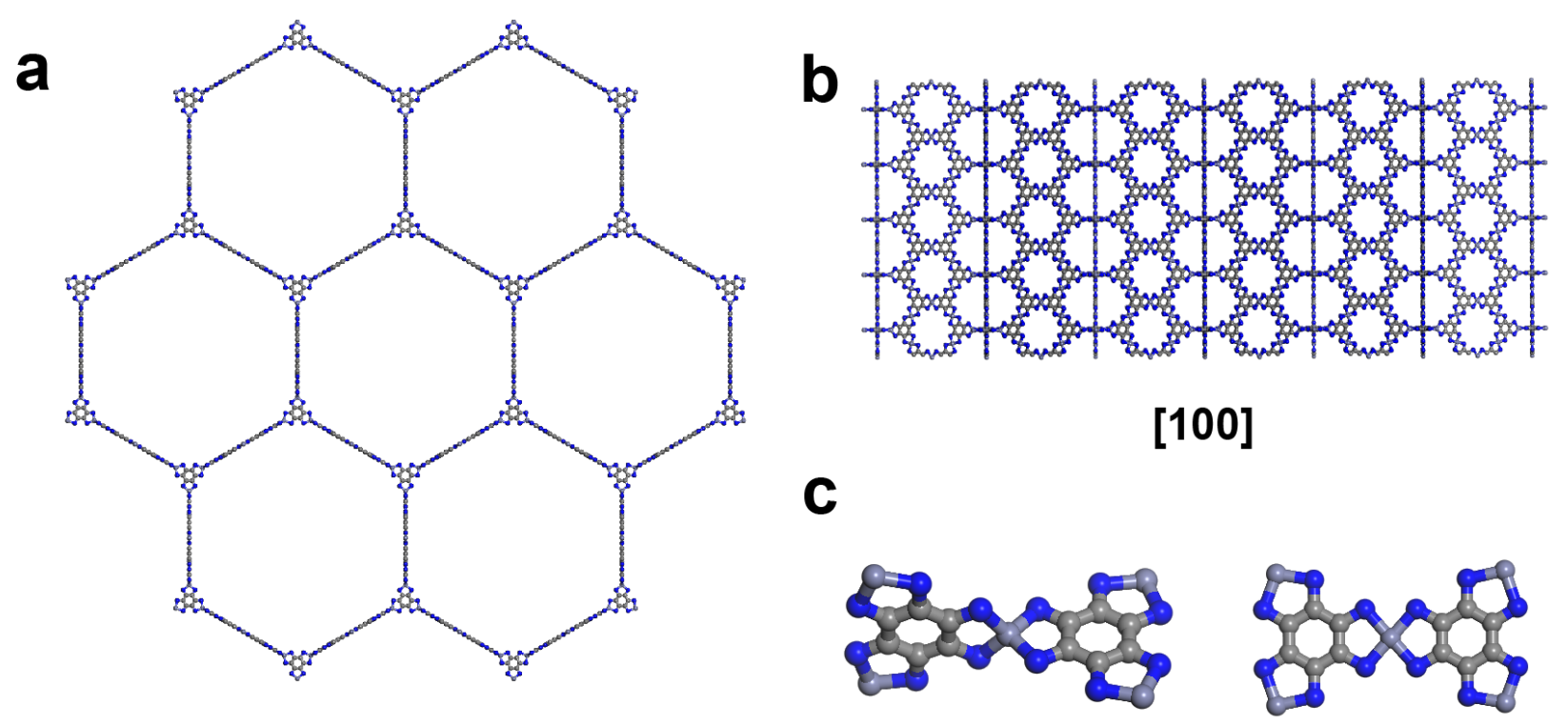

[001]

Figure S11 | Hypothetical model of Zn-HAB with a mixed tetrahedral and square planar coordination geometry of $\mathrm{Zn}$ atoms. (a and b) The structural model viewing along [001] and [100] directions, respectively. (c) The building units with tetrahedral and square planar Zn atoms.

\section{Section 4. Electrical measurements}

All electrical measurements were performed inside a LakeShore CRX-6.5K probe station at a pressure of $<2 \times 10^{-4}$ mbar. Zn-HAB pellets were cold pressed using commercial pressing equipment (MTI Corporation). The thickness of the pellet was measured with a micrometer (Mitutoyo). For electrical conductivity measurements, the pellet was affixed to a silicon wafer with double-sided polyimide tape. Carbon paste (Electron Microscopy Sciences) was used to glue one end of each of four gold nanowires to points evenly spaced around the pellet circumference and the other end of each to the substrate. The substrate was adhered to the temperature-controlled stage of the LakeShore probe station with silicone paste (Dow Corning). Four tungsten tip probes connected the carbon paste pads to a Keithley 4200-SCS parameter analyzer. A K-type thermocouple was affixed to the polyimide surface with additional silicone paste $1 \mathrm{~mm}$ from the pellet and the temperature monitored with an Omega Platinum temperature controller. $I-V$ curves were collected by scanning the current from -1.4 $\mu \mathrm{A}$ to $+1.4 \mu \mathrm{A}$ with a step size of $0.4 \mu \mathrm{A}$ between adjacent electrodes $\mathrm{A}$ and $\mathrm{B}$ and measuring the voltage drop across the opposite pair of electrodes, $\mathrm{C}$ and $\mathrm{D}$. A second $I-V$ curve was generated without rearranging any wires by passing current between $\mathrm{A}$ and $\mathrm{C}$ and measuring the voltage across $\mathrm{B}$ and $\mathrm{D}$. The electrical resistances at each temperature step were extracted from linear regression results of the $I-V$ curves. The conductivity as a function of temperature was calculated from the two resistances at each temperature and the pellet thickness according to the method discussed elsewhere. ${ }^{113}$ For Seebeck measurements, silicone thermal paste was used to attach a thin sheet of copper to an electrically isolated LakeShore triaxial sample holder. The bottom stage temperature was controlled with a Lakeshore Model 336 Cryogenic Temperature Controller. Pressed pellets were sandwiched between the bottom stage and a large copper block with temperature control achieved through a Peltier attached 
to an Omega Platinum controller (see Figure S12). The slit on one side of the copper block was filled with silicone thermal paste (Dow Corning) and a thin, leaf-type, K-type thermocouple (Omega Engineering) inserted into the slit. The voltage was measured at the opposite side through wiring soldered to a \#4-40 screw and attached via BNC to one of the probe station ports. The Seebeck voltage was measured using a Keithley 4200-SCS at eight temperature gradients around each set-point temperature $\pm 2 \mathrm{~K}$. The thermopower was determined by linear regression of the $V-\Delta T$ curve.
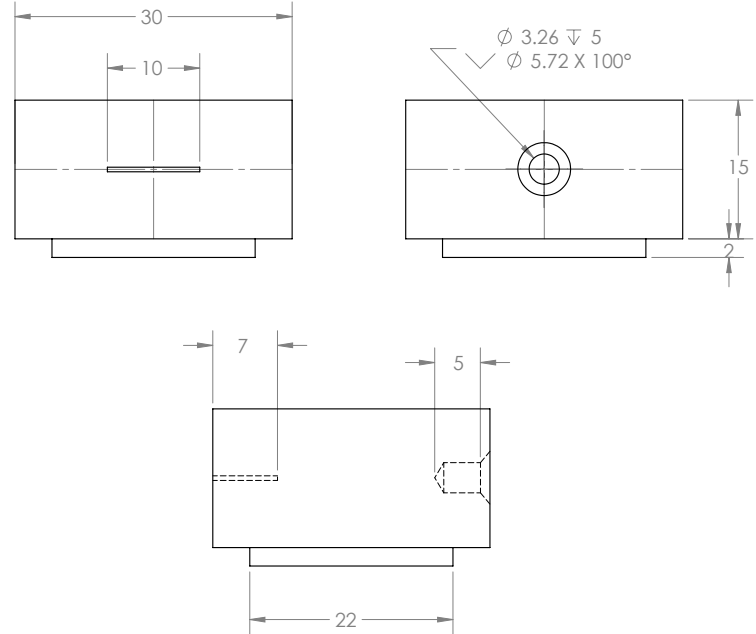

Figure S12 | Drawing of copper top contact for the cryostat.

\section{References}

S1. Mahmood, J.; Kim, D.; Jeon, I.-Y.; Lah, M. S.; Baek, J.-B. Syn. Lett. 2013, 24, 246-248.

S2. Coelho, A. A. J. Appl. Crystallogr. 2018, 51, 210-218. 